(C) 2019, American Psychological Association. This paper is not the copy of record and may not exactly replicate the final, authoritative version of the article. Please do not copy or cite without authors' permission. The final article will be available, upon publication, via its DOI:

$10.1037 / x h p 0000626$

\title{
Perceptual dissociations among views of objects, scenes, and reachable spaces
}

\author{
Emilie Josephs and Talia Konkle \\ Department of Psychology, Harvard University, Cambridge, MA, USA
}

Correspondence concerning this article should be addressed to Emilie Josephs, Department of Psychology, Harvard University, Cambridge, MA 02143. Contact: ejosephs@g.harvard.edu

Word count $=7078$ 


\begin{abstract}
In everyday experience, we interact with objects and we navigate through space. Extensive research has revealed that these visual behaviors are mediated by separable objectbased and scene-based processing mechanisms in the mind and brain. However, we also frequently view near-scale spaces, e.g. when sitting at the breakfast table or preparing a meal. How should such spaces (operationalized here as "reachspaces"), which contain multiple objects but not enough space to navigate through, be considered in this dichotomy? Here we used visual search to explore the possibility that reachspace views are perceptually distinctive from full-scale scene views, as well as object views. In the first experiment, we found evidence for this dissociation. In the second experiment, we found that the perceptual differences between reachspaces and scenes were substantially larger than those between scene categories (e.g. kitchens vs. offices). Finally, we provide computational support for this perceptual dissociation: deep neural network models also naturally separate reachspaces from both scenes and objects, suggesting that mid-to-high level features may underlie this dissociation. Taken together, these results demonstrate that our perceptual systems are sensitive to systematic visual feature differences that distinguish objects, reachspaces, and full-scale scene views. Broadly, these results raise the possibility that our visual system may use different perceptual primitives to support the perception of reachable and navigable views of the world.
\end{abstract}

Keywords: visual search, visual features, reachspaces, scenes, objects, space 


\section{Public Significance Statement}

The present study suggests that views of near-scale space (e.g.: kitchen counters, office desktops) look systematically different than views of far-scale space (e.g.: full scale kitchens, full scale offices). These perceptual differences were substantially larger than those between scene categories (e.g. kitchens vs. offices). 
Title: Perceptual dissociations among views of objects, scenes, and reachable spaces

As we behave in the world, there is a clear distinction between spatially compact elements of the environment that we can hold, and spatially extended elements that we can move through; namely, between objects and scenes (Epstein, 2005; Henderson \& Hollingworth, 1999). Extensive evidence from development, neuropsychology, and cognition research suggests that this fundamental division is reflected in our cognitive architecture, with different developmental trajectories, disorders and processing demands associated with each (e.g. Spelke, 1990; Carey \& $\mathrm{Xu}, 2001$; Henderson \& Hollingsworth, 1999; Carey, Dijkerman, Murphy, Goodale, \& Milner, 2006; Epstein, DeYoe, Press, Rosen, \& Kanwisher, 2001; Landis, Cummings, Benson \& Palmer, 1986; Steeves, Humphrey, Culham, Menon, Milner, \& Goodale, 2004). This distinction is also evident in the visual processing stream, where distinct brain regions are sensitive to object-based and scene-based perceptual properties, and support object- and scene-related processing respectively (e.g. Grill-Spector, Kourtzi \& Kanwisher, 2001; Biederman, 1987; Epstein \& Kanwisher, 1998; Greene \& Oliva, 2009, 2010).

However, real-world views of objects and scenes exist along a continuum: between the close-up view of a carrot and the far-scale view of the kitchen is the near-scale view of the countertop where you prepared the food. Consider also the view of the desk as you type an email, or a workbench as you solder a wire, or a place-setting as you eat a meal. These views, which we here refer to as "reachspaces" (see Figure 1), are like scenes, in that they extend beyond the view and contain multiple objects. But unlike scenes, you do not navigate your body
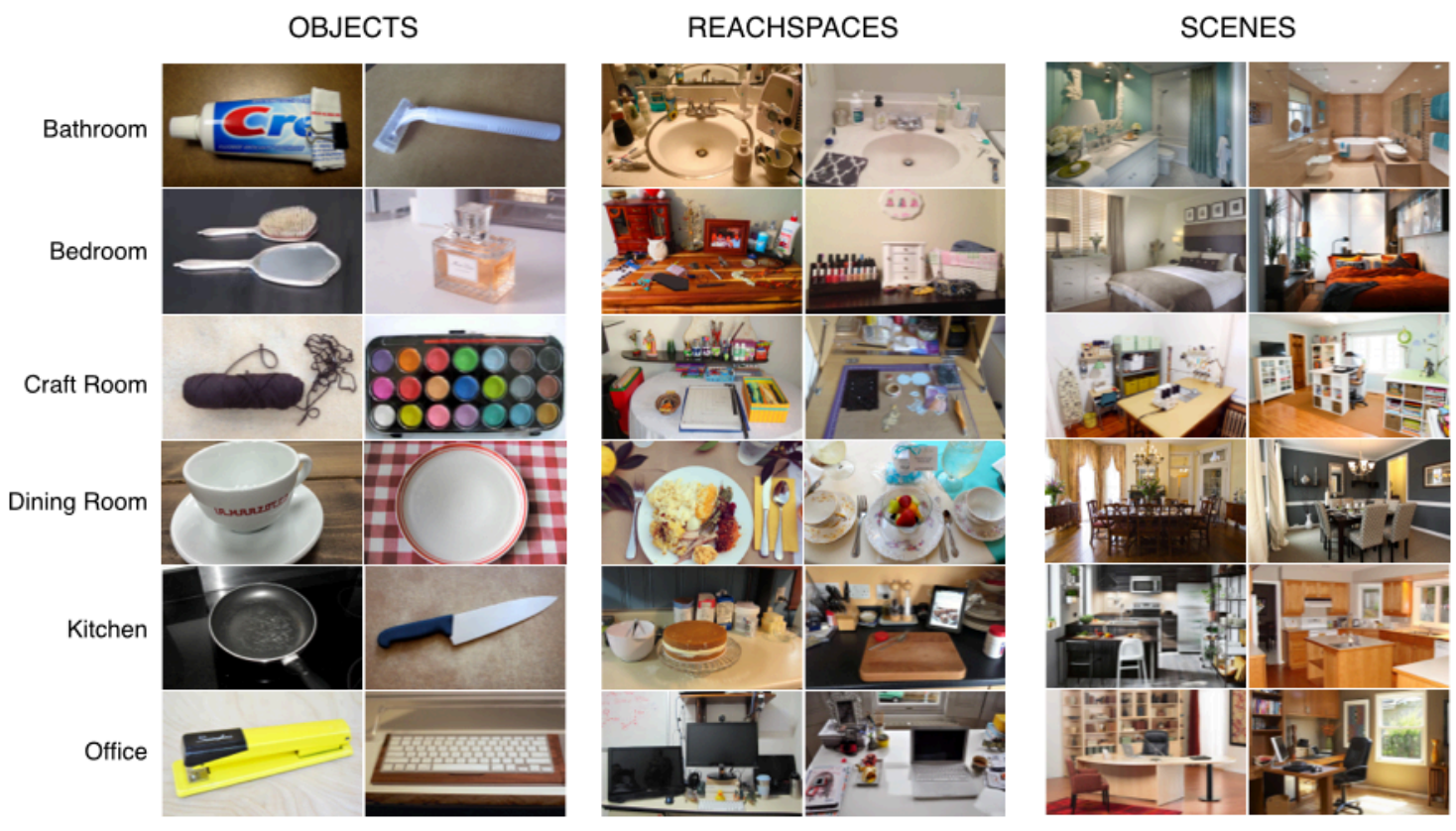

Figure 1. Examples of the Object, Reachspace, and Scene stimuli. Object images consisted of single objects on their natural backgrounds. Reachspace images consisted of close-scale spaces, delineated by a horizontal surface and filled with objects, where everyday tasks are typically performed. Scene images consisted of full views of indoor rooms. 
through them; instead, the interaction demands are more object-like in that they involve coordinated hand actions.

Such intermediate views have historically been treated as equivalent to navigable-scale scenes (Intraub, 2010), or have been acknowledged as an uneasy fit in the object/scene dichotomy, and then consequently omitted from scene perception research (c.f. Henderson \& Hollingworth, 1999). However, this omission may have obscured important distinctions, as previous work suggests that the ways in which we act, remember and deploy attention may differ for near and far space. For example, boundary extension, the anticipatory representation of the space beyond the edge of an image, is stronger for near space than far space (Bertamini, Jones, Spooner \& Hecht, 2005; Intraub, Bender \& Mangels, 1992). Additionally, attention can be impaired for spaces near the body but not farther way, and vice versa, in patients with hemispatial neglect (Cowey, Small \& Ellis, 1994; Halligan \& Marshall, 1991). Finally, evidence from the visuomotor literature highlights that the distinction between what is in and out of reach is a prominent one, with near-space coding evident extensively across dorsal stream regions (e.g. Gallivan et al., 2009; Maravita \& Iriki, 2004). Thus, there is both behavioral and neural evidence that there may be important differences in the processing of near and far space.

In the present study, we asked whether these functional differences between scales of space run alongside perceptual differences in their visual appearance. Specifically, we tested whether there are systematic perceptual differences between reachspaces and scenes. On one hand, both reachspaces and scenes depict extended surfaces with multiple objects, and thus may rely on common perceptual features. Consistent with this reasoning, some studies have used views of reachable spaces to highlight mechanisms of scene perception, with the assumption that near and far-scale views can be used interchangeably (e.g. Võ \& Wolfe, 2013; Epstein, Graham \& Downing, 2003). On the other hand, reachspaces contain small objects while full scenes views are dominated by large objects, and small and large objects have dissociable perceptual features (Long, Konkle, Cohen, \& Alvarez, 2016). There may also be differences in their global layout features, as previous computational models have leveraged natural image statistics to estimate the depicted depth in an image along the full object-scene continuum (e.g. Torralba \& Oliva, 2002; Torralba \& Oliva, 2003). These results raise the possibility that there are different image statistics for near- and far-scale space, which human perceptual systems may be sensitive to, enabling views of reachspaces to dissociate from views of scenes in perception.

We also examined whether reachspaces views perceptually dissociate from object views. While objects are bounded entities, images of objects on a naturalistic background must necessarily depict some space, leading to some ambiguity with reachspace views. However, it is possible to operationalize the differences between these two kinds of views in terms of their implied viewing distances (c.f. Intraub 2010, 2012). Object views gives a sense of a very close viewing distance ( $~ 8-12$ inches away), and feature a central object on a homogeneous background, cropped so that it fills much of the image, with minimal to no "layout" edges, e.g. where a counter meets a back wall or a corner. In contrast, reachspaces views convey a sense of the space about of 3-4 feet from the viewer, have salient 3-D layout features (e.g. a flat horizontal surface and a back wall), and contain an array contextually related objects. Object views are scaled such that a hand would fill the frame while reachspace views are scaled such that both arms would fit in the space. Thus, object and reachspace views are sampled from systematically different views on the environment, and as such, they may have different visual feature properties. 
To examine whether reachspaces are perceptually distinct from scenes and objects, we employed both behavioral and computational methods. First, we used a visual search paradigm, where the speed of search depends on how visually distinctive the target is from the distractors (Duncan \& Humphreys, 1989; Wolfe \& Horowitz, 2017). Across several experiments, we found that reachspaces systematically dissociate from both full-scale scenes and singleton objects in search displays, with an effect that is substantially larger than the perceptual difference between semantic categories of scenes. Second, examining deep convolutional neural networks trained to perform either object or scene recognition, we found that both kinds of networks naturally distinguish reachspace views from both objects and scenes in middle and later layers. This result provides computational support for the existence of a distinctive visual representation of reachspaces, and begins to address questions about the nature of these feature distinctions. Broadly, these results raise the possibility that there may be separate perceptual processing mechanisms for reachable and navigable space.

\section{Experiment 1: A 3-way dissociation in visual search performance}

To explore the possibility that reachspaces perceptually dissociate from objects and scenes, we used a visual search paradigm (e.g. following Cohen, Alvarez, Nakayama \& Konkle, 2017). Under the logic of a visual search task, targets that are different from distractors will stand out, and will be faster to find in a search array than targets that are similar (Duncan \& Humphreys, 1989). Thus, for example, if reachspaces are perceptually distinct from scenes, then it should be easier to find a reachspace among scenes than among other reachspaces. Visual search speeds are strongly influenced by visual similarity, (e.g. leveraging feature differences between line orientations, curvature, and shape), and are largely unaffected by non-visual (semantic) information in the displayed items (see Wolfe \& Horowitz, 2017). Thus, any differences in visual search times that we find between objects, reachspaces, and scenes, would provide evidence for a dissociation at the level of visual perception. Experiment 1 tests this hypothesis on two image sets: images in Expla are matched in luminance and contrast, while images in Exp1b are additionally matched in global spatial frequency content. This image set manipulation enables us to test for visual differences over and above relatively primitive global image statistics.

\section{Methods}

Participants. 44 participants were enrolled $(\mathrm{N}=22$ each in Experiment $1 \mathrm{a}$ and Experiment $1 \mathrm{~b}$ ). These sample sizes were estimated to provide $80 \%$ power using a simulation method (see Appendix for details). Demographic information was not recorded from individual participants, but all participants were between the ages of 18 and 35, and were recruited from a participant population that consisted of $65 \%$ women. All participants gave informed consent and were compensated with $\$ 10$ or class credit for their participation. All procedures were approved by the Harvard University Human Subjects Institutional Review Board.

Stimuli. The stimulus set consisted of views of objects, reachspaces, and scenes (examples in Figure 1). Each of these image scales contained 12 images from each of 6 semantic categories (bathroom, bedroom, craft room, dining room, kitchen, office), yielding 72 images per scale. Object images depicted close-scale views (within 8-12 inches from the object) of single objects on their natural background, e.g.: a close-up view of a sponge with a small amount of 
granite countertop visible beyond it. Reachspace images depicted near-scale spaces that were approximately as deep as arm's reach (3-4ft), consisting of multiple small objects arrayed on a horizontal surface, e.g.: a knife, cutting board and onion arrayed on kitchen counter. Scene images depicted full views of the interior of rooms, e.g.: a view of a home office. Images were collected from Google Images under fair-use, and were scaled to a resolution of $800 \times 1280$ pixels. All stimuli are available for download on the Open Science Framework (osf.io/7j6cx).

Images were controlled using the SHINE toolbox (Willenbockel, et al, 2010), to be matched in their average luminance (Experiment 1a), and in both average luminance and in spatial frequency (Experiment 1b). Examples are shown in Figure 2b. Images for Experiment 1a were luminance matched using the lumMatch function run with the default settings, and images for Experiment 2 were spatial frequency-matched using the specMatch function, then luminancematched using the histMatch function, both with default parameters.

Design. Participants searched for a single target in an array of distractors (Figure 2a). Each trial started with a fixation cross in the center of the screen for $500 \mathrm{~ms}$. Next, a preview of the target appeared in the center of the screen for 500ms. Following a second 500ms fixation screen, the search display appeared, consisting of 6 images arranged in a circle to be equidistant from the center of the screen. No images were placed on the vertical midline. One of these images was always the target. Participants pressed the spacebar when they found the target, and then all images in the display were replaced by X's. Participants clicked on the X corresponding to the target location. For correct responses, the next trial would start after a variable time interval lasting between $500 \mathrm{~ms}$ and $1000 \mathrm{~ms}$, but for incorrect responses, participants received a feedback message for three seconds before the next trial began.

The experiment was a $3 \times 3$ design: on a given trial, a target could be an object, a reachspace, or a scene, and could be displayed among distractors that were all objects, all reachspaces or all scenes, leading to a fully-crossed design with a total of 9 conditions. There were 50 trials in each of the 9 conditions. On every trial, targets and distractors were randomly selected from the appropriate image scale, with the constraint that none of them could have the same semantic category as the target. Thus, for example, a kitchen reachspace target could appear among scene distractors that included bathrooms, bedroom, craft rooms, dining rooms or offices, but not kitchens. The experiment began with 10 practice trials (practice images were drawn from the same set used in the experimental trials), followed by 9 blocks of 50 experimental trials.

Apparatus. Experiments were run on a 24-inch iMac running OS X 5.10.8 in MATLAB 7.10.0 (The MathWorks, Natick, MA) using the Psychophysics Toolbox (Brainard, 1997; Kleiner, Brainard, Pelli, 2007; Pelli, 1997). The monitor was set to a spatial resolution of 1,920 $\times$ 1,200 pixels and a refresh rate of $60 \mathrm{~Hz}$. Observers were seated approximately $57 \mathrm{~cm}$ from the monitor, so $1 \mathrm{~cm}$ on the screen subtended $1^{\circ}$ of visual angle. All images were shown at a size of $8^{\circ}$ by $5^{\circ}$ visual angle ( $300 \times 188$ pixels). Responses were recorded on a standard Apple Keyboard.

Data Analysis. Reaction time (RT) data were trimmed to remove outliers using the following procedure. First, incorrect trials and trials with RTs less than $200 \mathrm{~ms}$ were excluded. Mean RT was then calculated for each condition and each participant, and RTs that fell more than 3 standard deviations away from the mean (for a given participant on a given condition) 
were discarded (Rousseeuw \& Croux, 1993). RTs were log transformed prior to trimming to account for the fact that reaction time distributions are right skewed (Ratcliff, 1979; Palmer, Horowitz, Torralba \& Wolfe, 2011). This procedure led to the exclusion of $1.2 \%$ of trials from Experiment $1 \mathrm{a}$ and $0.6 \%$ of trials from Experiment $1 \mathrm{~b}$. Individual participants who lost more than $15 \%$ of their trials to this trimming were replaced. One subject was dropped from Exp 1a for this reason and replaced, and no subjects were dropped from Exp 1b. Planned pairwise one-tailed t-tests were used to assess statistical significance. Effect size was calculated using the classical Cohen's $d$ : the difference between the means of the conditions divided by the pooled variance.

\section{Results \& Discussion}

Figure 2c shows the results of the visual search experiments, using luminance-matched images (Exp 1a) and spatial-frequency matched images (Exp 1b). To interpret the pattern of results across the 9 conditions ( 3 target view types $\mathrm{x} 3$ distractor view types), we examined the conditions in pairs: e.g. if object are perceptually distinct from scenes, they should be found faster in displays of scene distractors than in displays of object distractors. Indeed, as expected, objects and scenes dissociate from each other: object targets were found more quickly among scenes than among other objects, $(\operatorname{Exp} 1 \mathrm{a}: t(21)=4.59, \mathrm{p}<0.001, d=0.52$; Exp 1b: $t(21)=$ $4.20, \mathrm{p}<0.001, d=0.48)$. In the complementary comparison, scenes targets were likewise found more quickly among objects than among other scenes (Exp 1a: $t(21)=13.93, \mathrm{p}<0.001, d=2.33$; Exp 1b: $t(21)=12.37, \mathrm{p}<0.001, d=1.98)$.

In the critical comparisons, we found that reachspaces dissociated from scenes. That is, reachspaces targets were found faster among scenes than among other reachspaces (Exp 1a: $t(21)$ $=2.09, \mathrm{p}=0.024, d=0.22 ; \operatorname{Exp} 1 \mathrm{~b}: t(21)=3.04, \mathrm{p}=0.003, d=0.34)$, and likewise scenes targets were found faster among reachspaces than other scenes (Exp 1a: $t(21)=3.92, \mathrm{p}<0.001$, $d=0.62$; Exp 1b: $t(21)=5.64, \mathrm{p}<0.001, d=0.74)$. Additionally, we found that reachspaces dissociated from objects: reachspace targets were found more quickly among objects than among other reachspaces $(\operatorname{Exp} 1 \mathrm{a}: t(21)=8.52, \mathrm{p}<0.001, d=1.02$; Exp 1b: $t(21)=7.51, \mathrm{p}<0.001, d=$ 0.92 ), and correspondingly object targets were found more quickly among reachspaces than among other objects $(\operatorname{Exp} 1 \mathrm{a}: t(21)=1.74, \mathrm{p}=0.048, d=0.21 ; \operatorname{Exp} 1 \mathrm{~b}: t(21)=3.18, \mathrm{p}=0.002, d$ $=0.35)$.

These results, replicated across two experiments, provide evidence that reachspaces are perceptually different from both full-scale scenes and singleton objects. Given that both reachspaces and scenes have a spatial layout and consist of multiple objects, it could have been the case that they are visually encoded using the same perceptual features. However, the visual search data instead suggest that the perceptual content of views of reachable space is systematically different from the perceptual content of full-scale scenes. Furthermore, these behavioral dissociations persist in spatial-frequency matched images (Exp 1b), indicating that differences in global spatial frequency or luminance content are not solely responsible for distinguishing reachspaces from objects and scenes. Finally, these data do not require that objects, reachspaces and scene be separate categories in the mind a priori; it is possible that participants could perform this task using on-the-fly categories developed in the context of this particular visual search design. However, critically, the main conclusion that there are perceptual feature differences between these three scales does not depend on the exact strategy used in performing the task. 
A.

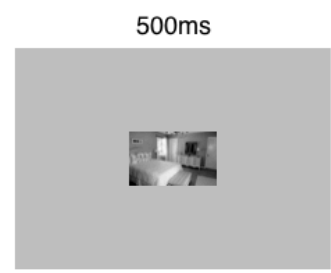

500ms

until button press
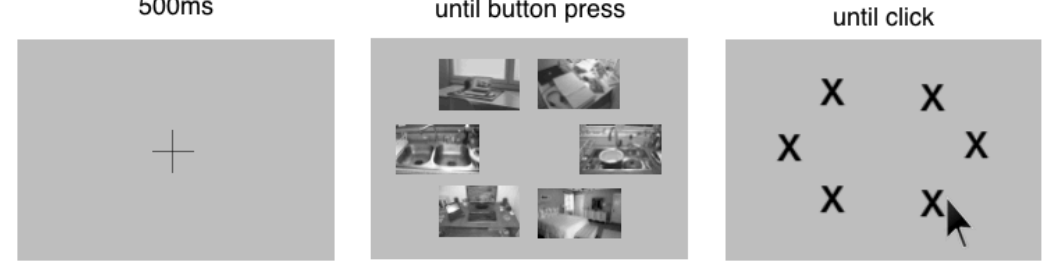

time

B.
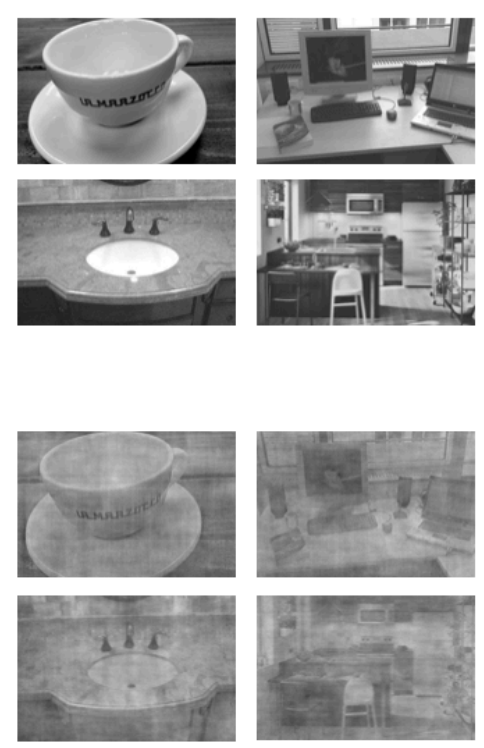

C.
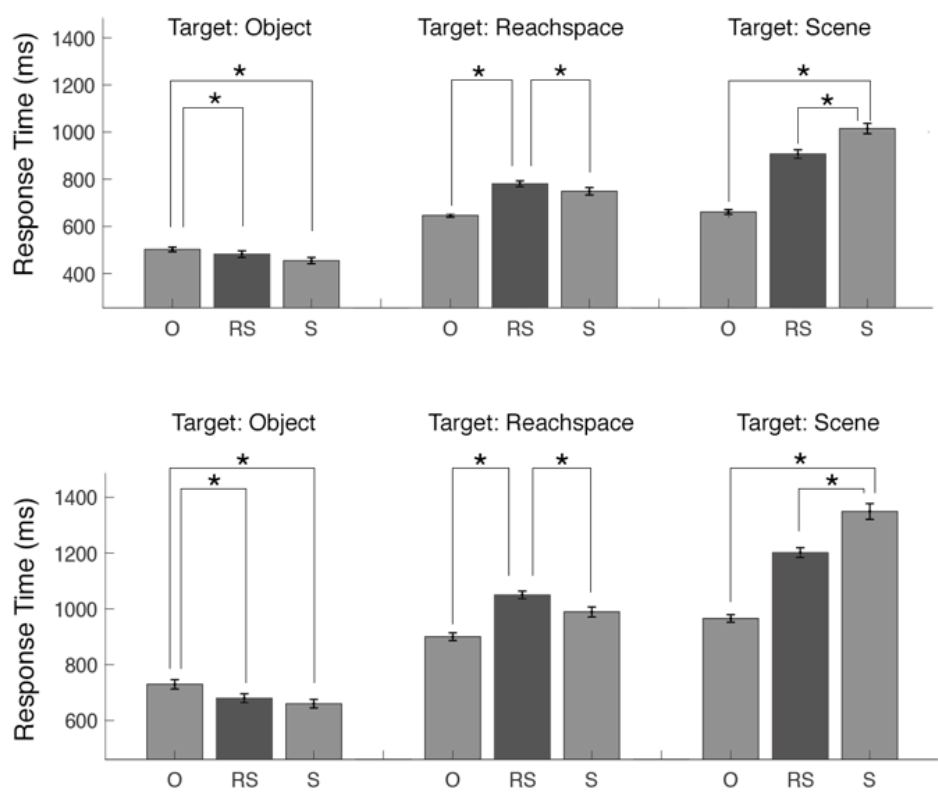

Figure 2. Trial design, stimuli and results for Experiment 1. A. Example time course of a single trial. A target was presented for $500 \mathrm{~ms}$, followed by a $500 \mathrm{~ms}$ blank, followed by the search display. The search display contained 6 items, one of which was the target. Participants pressed the space bar when they found the target, replacing images by Xs, then clicked the location of the target with the mouse. B. The stimuli for Experiment 1a (top) and $1 \mathrm{~b}$ (bottom). Images in Experiment 1a were matched in mean luminance. Images in Experiment $1 \mathrm{~b}$ were matched in both luminance and spatial frequency. C) Reaction time results for Experiment 1a (top) and $1 \mathrm{~b}$ (bottom). Reaction times is plotted for each target-distractor combination, with the first set of bars reflecting Object targets, the second set of bars reflecting Reachspace targets, and the third set of bars reflecting Scene targets. Stars mark significant differences. Error bars show within-subject standard error of the mean (Morey, 2008).

In addition, broader trends in the data shed some light on the nature of this 3-way dissociation, suggesting that reachspaces are perceptually intermediate. That is, the reaction time differences imply that objects and scenes are the most perceptually dissimilar, and that reachspaces come somewhere in between: scenes were found more quickly among objects than among reachspaces (Exp 1a: $t(21)=13.02, \mathrm{p}<0.001, \mathrm{~d}=1.68$; Exp 1b: $t(21)=11.32, \mathrm{p}<0.001, \mathrm{~d}$ $=1.44$; post hoc paired one-sided t-test). Likewise, objects were found more quickly among scenes than among reachspaces $(\operatorname{Exp} 1 \mathrm{a}: t(21)=5.55, \mathrm{p}<0.001, \mathrm{~d}=0.35$; Exp $1 \mathrm{~b}$ did not reach significance: $t(21)=1.27, \mathrm{p}=0.106, \mathrm{~d}=0.14)$. Additionally, we find further evidence that reachspaces are perceptually intermediate when considering overall search times by target: object targets overall were found faster than reachspaces, which were found faster than scenes 
(Expla main effects: $\mathrm{O}=480 \mathrm{~ms} ; \mathrm{RS}=726 \mathrm{~ms} ; \mathrm{S}=861 \mathrm{~ms} ; 3 \times 2$ ANOVA main effect of scale $F(2,194)=111.38, \mathrm{p}<0.001$; Exp1b main effects: $\mathrm{O}=689 \mathrm{~ms} ; \mathrm{RS}=979 \mathrm{~ms} ; \mathrm{S}=1172 \mathrm{~ms} ; 3 \times 2$ ANOVA main effect of scale $F(2,194)=119.36, \mathrm{p}<0.001)$. Taken together, these visual search dissociations demonstrate that reachspaces are perceptually distinguishable from, and intermediate to, objects and scenes.

\section{Experiment 2: Pitting the Effect of Image Scale Against Scene Category}

How substantial are the perceptual differences between reachspaces and full-scale scenes? Will any meaningful distinction among visual environments give rise to similar visual search effects, or do the present dissociations constitute a particularly sizeable difference? To put the perceptual dissociation between reachspaces and scenes into context, we compared it to an alternative distinction that is critical for scene processing: semantic category. It is clear that the visual system is sensitive to perceptual features that help distinguish among scene categories: within a brief glance, observers can readily identify the semantic category of a scene, e.g. whether it is a kitchen or an office (Potter, 1975; 1976). The aim of Experiment 2 was to assess the magnitude of scene-category effects on visual search time and compare this to the magnitude of the reachspace-scene effect. Specifically, we tested whether searching for a scene target was faster when the distractors differed by scene category, by scale of space, or both (Figure 3a).

\section{Methods}

Participants. 27 participants were enrolled in Experiment 2. This sample size was estimated to provide $80 \%$ power according to the same simulation used for E1 (see Appendix). As before, participants were between the ages of 18 and 35, and had normal or corrected to normal vision. All participants gave informed consent, were recruited from the Harvard Psychology Department participant pool, and were compensated with $\$ 10$ or class credit for their participation. All procedures were approved by the Harvard University Human Subjects Institutional Review Board.

Stimuli. The stimulus set consisted of 72 images of scenes from 6 categories and 72 images of reachspaces from 6 categories (this constituted all the scene and reachspaces images from Experiment 1a). These images were matched in average luminance.

Design. Participants searched for a target among distractors with the same trial timing and display parameters as in Experiment 1, but with different target-distractor conditions (Figure 3a). The target in this experiment was always a scene image. The distractors could be either reachspaces or scenes, drawn from either the same or different semantic category as the target scene. There were 30 trials in each of these four conditions (same scale / same categ; diff scale / same categ; same scale / diff categ; diff scale / diff categ). To counterbalance scene category, scene targets were drawn equally from the 6 semantic categories, appearing 5 times each in each condition. For conditions where distractors were from a different semantic category than the target, these were balanced such that each target category appeared among each of the other 5 distractor categories exactly once. Trials were split into two blocks of 60 trials, with 11 practice trials at the beginning. Trial order was randomized over the experiment.

Data Analysis. The data trimming procedure was the same as in Experiment 1. This procedure led to the exclusion of $2.0 \%$ of trials from Experiment 2. One subject was dropped 
from Exp 2 for losing more than 15\% of their trials to trimming, and was replaced. Planned pairwise one-tailed t-tests were used to assess statistical significance, and Cohen's $d$ was used to estimate effect size as described in Experiment 1.

\section{Results \& Discussion}

Average reaction times for Experiment 2 are shown in Figure 3b. Search was slowest when the target scene matched the distractors in both category and scale (e.g. a bathroom scene among other bathroom scenes; $1290 \mathrm{~ms}, \mathrm{SEM}=29 \mathrm{~ms}$ ), establishing a baseline condition from which to compare the other conditions. When distractors differed from the target in their semantic category alone (e.g., an office scene among dining room scenes), search was significantly faster than the baseline $(130 \mathrm{~ms}$ faster, $t(26)=3.51 \mathrm{p}=0.001, d=0.57)$. This result confirms that different scene categories are perceptually dissociable. Search was also significantly faster than baseline when the distractors differed in scale (e.g., an office scene among office reachspaces: $225 \mathrm{~ms}$ faster than baseline, $t(26)=6.42, \mathrm{p}<0.001, d=1.02)$. This result confirms that reachspaces are perceptually dissociable from scenes. Crucially, the image scale effect was much larger than the semantic category effect $(130 \mathrm{~ms}$ vs. $225 \mathrm{~ms}$; post hoc paired one-sided t-test: $t(26)=3.15, \mathrm{p}=0.002, d=0.51)$. Finally, there was no additional speed to be gained when distractors differed in both image scale and scene category ( $9 \mathrm{~ms}$ difference between reachspace distractors of the same vs different semantic category, $t(26)=0.51, p=0.31$, $\mathrm{d}=0.05)$.

These results reveal that the perceptual distinction between reachspaces and scenes has a substantially larger impact on visual search behavior than perceptual distinctions between semantic categories. Further, these data serve as a replication of one of the key results in Experiment 1: reachspaces are not "just scenes" when it comes to visual search behavior.

A.

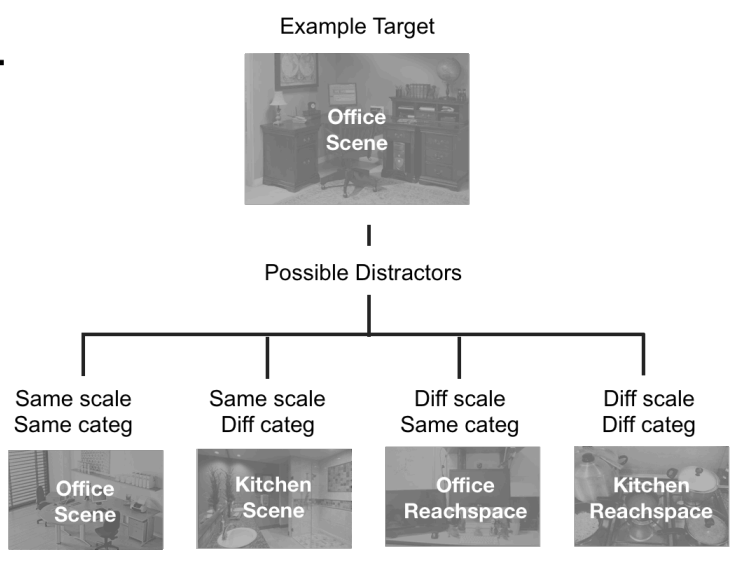

B.

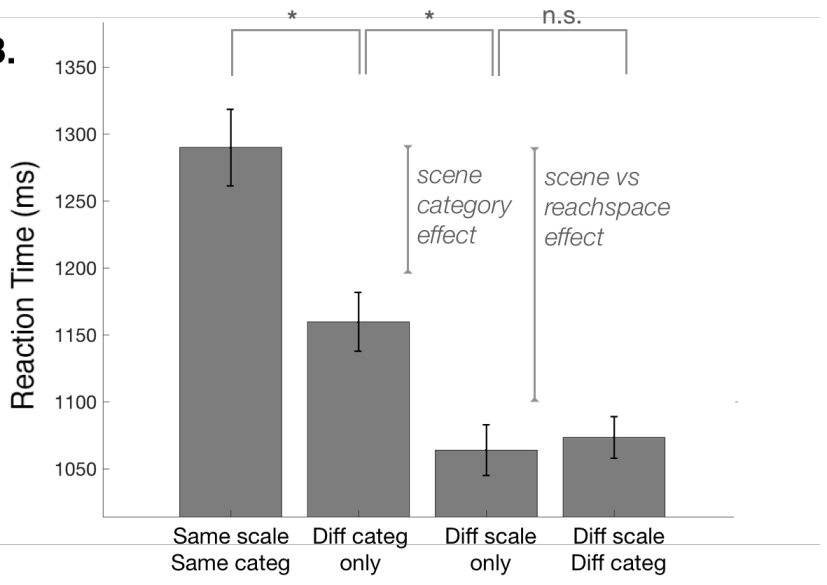

Figure 3. Trial Design and results for Experiment 2. A) The four conditions in Experiment 2. The target in each trial was always a scene image, drawn from one of six semantic categories. These targets were shown among distractors that could be either of the same or different image scale and the same or different semantic category. B) Reaction times are plotted for the four conditions: same view type same category; same view type different category; different view type different category; different view type different category. Error bars represent within-subject standard error of the mean (Morey, 2008). 


\section{Visual Feature Analysis: Three-way Dissociation in Computational Image Features}

To this point, we have used reaction time-based behavioral tasks to infer that our perceptual systems are sensitive to systematic differences among different views of space. Such perceptual differences must arise from different image-computable visual statistics present in each of the views. Thus, we next turned to a computational modeling approach to bolster this claim with a computational existence proof of such feature differences, and to provide some insight into the possible nature of these visual feature distinctions. Specifically, we used deep convolutional neural networks, which can be treated as sophisticated pattern extractors, to measure visual feature differences among the views. Furthermore, since these models measure purely visual information - divorced from semantic interpretation, prior information or expectations - they provide a strong test of the hypothesis that the three scales of space explored here differ in their visual content.

DNNs are currently the state-of-the-art for object and scene recognition by computer vision systems. Through extensive exposure to natural images, these models learn to detect particular visual features from an image in order to perform a specified task (e.g. object categorization, scene categorization). Critically, feature detector neurons are arranged in hierarchical layers, where early layers detect simpler image statistics and deeper layers detect increasingly complex features, based on weighted combinations of the features in the previous layer. Currently, the features learned by DNNs are the best model for predicting biological feature tuning along the visual processing stream, outperforming categorical models and other visual feature models (Cadieu, Hong, Yamins, Pindo, Ardila, Solomon, Majaj \& DiCarlo, 2014; Cichy et al, 2017; Güçlü \& van Gerven, 2015; Khaligh-Razavi \& Kriegeskorte, 2014; Yamins, Hong, Cadieu, Solomon, Seibert \& DiCarlo, 2014), and can account for some structure in behavioral judgments of objects similarity (Jozwik, Kriegeskorte, Storrs \& Mur, 2017; King, Groen, Steel, Kravitz \& Baker, 2018). This correspondence between models, brains, and behavior is especially noteworthy because these DNN models were not optimized to predict brains or behavioral similarity judgments, suggesting that artificial and biological systems have arrived at similar solutions of what visual features are useful for distinguishing among categories, even if learned in very different ways. Thus, deep neural networks provide a powerful tool for exploring visual feature dissociations using an artificial visual system (e.g. Bonner \& Epstein, 2018; Groen et al, 2018).

The logic of our approach was to compute deep net feature responses to the images in the stimulus set, and then test whether reachspaces dissociate from objects and scene images (Figure 4). We considered two different pre-trained neural networks. The first model was a 7-layer AlexNet architecture trained to perform object recognition (Krizhevsky, Sutskever \& Hinton, 2012), henceforth, "ObjectNet", and the second model had the same architecture, but was trained to perform scene recognition ("SceneNet"). By comparing these two networks, we can examine whether any distinctions between objects, reachspaces, and scenes depend on features learned in service of object-category or scene-category distinctions. For each layer in each model, we calculated the response of each feature detector to each image in the set. By comparing how well objects, reachspaces, and scenes dissociate in each layer, we can infer something about the complexity of any features that distinguish these classes. Finally, we repeated this process for original full-colored stimuli, as well as the controlled stimuli of Experiment 1b. By comparing 


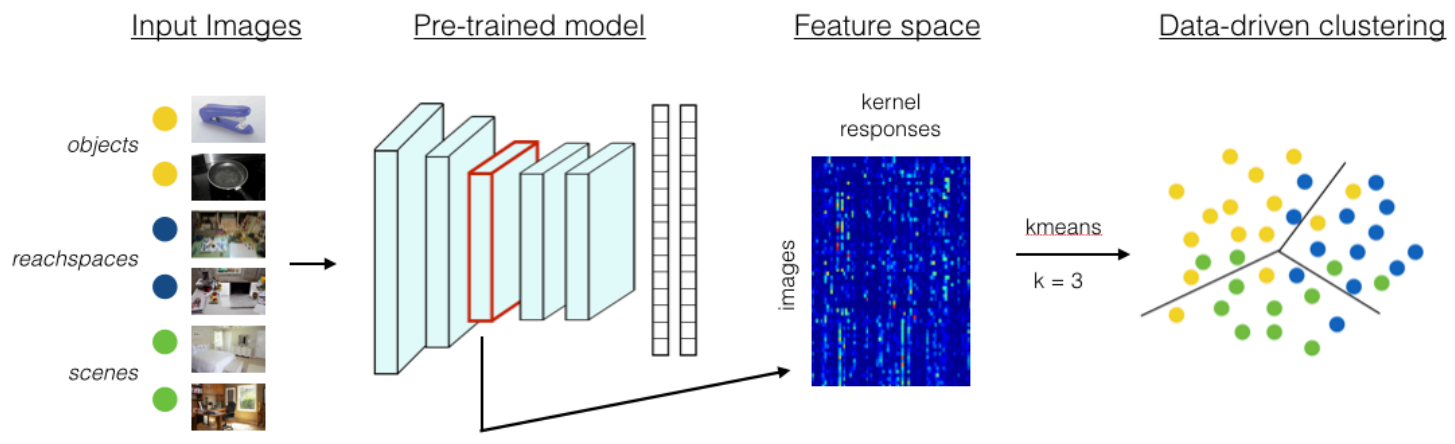

Figure 4. Extracting deep neural network features. We assessed whether data-driven clustering performed over the features could recapitulate the division between the three scales of space. Feature were extracted for each layer of a pre-trained deep neural network. For a given layer, kernel activations were extracted for every image in the set, then submitted to k-means clustering with $\mathrm{k}=3$, where images were assigned to a cluster based on their feature signatures. We then measured the correspondence between the clustering solution and the groundtruth grouping of the images by scale of space.

original and controlled image sets, we can verify that any dissociations persist across changes in low-level features, as they did in human visual search behavior.

\section{Methods}

Deep Neural Networks. For each DNN, we used an AlexNet architecture (Krizhevsky, Sutskever \& Hinton, 2012). Images were input at size $224 \times 224 \times 3$ pixels. Layer 1 was a convolutional layer with 64 kernels of size $11 \times 11 \times 3$ with a stride of 4 pixels and padding of 2 pixels. Layer 2 was a convolutional layer with 192 kernels of size $5 \times 5 \times 64$ with a stride of 1 pixels and padding of 2 pixels. Layer 3 was a convolutional layer with 384 kernels of size $3 \times 3 \times 192$ with a stride of 1 pixels and padding of 1 pixel. Layer 4 was a convolutional layer with 256 kernels of size $3 \times 3 \times 384$ with a stride of 1 pixel and padding of 1 pixel. Layer 5 was a convolutional layer with 256 kernels of size $3 \times 3 \times 256$ with a stride of 1 pixel and padding of 1 pixel. Layer 6 and 7 were fully-connected layers of 4096 neurons each.

One instantiation of this architecture was trained to do 1000-way object categorization on the ImageNet database (Russakovsky*, Deng*, Su, Krause, Satheesh, Ma, Huang, Karpathy, Khosla, Bernstein, Berg and Fei-Fei, 2015). A second instantiation of this architecture was trained to do 205-way scene categorization using the Places database (Zhou, Lapedriza, Xiao, Torralba, \& A. Oliva, 2014). Both networks were built and trained using in-lab software.

The standard AlexNet training regime was adopted using a public code package (https://github.com/soumith/imagenet-multiGPU.torch) that was optimized for multi-threaded CNN training in Torch7. Specifically, stochastic gradient descent (SGD) optimization was used with 0.9 momentum, an initial learning rate of 0.02 , and weight decay of 0.0005 . Both the learning rate and the weight decay follow a pre-defined decreasing schedule using a mini-batch size of 128, with 10,000 mini-batches per epoch over a total of 55 training epochs. Standard data augmentation such as random horizontal flips and random 224-by-224 crops were performed during training. 
Stimuli. The image set was the same as in Experiment 1, cropped and resized to $224 \times 224$ pixels to match the expected input size of Layer 1. Neural net activations were recorded for fullcolor versions of the images ("original"), as well as luminance and spatial frequency-controlled versions ("controlled").

Feature Extraction. After training on either ImageNet or Places205, DNN weights were frozen, and the activations to our stimulus images were measured. Image features were extracted separately from each layer of each network using the following procedure. For convolutional layers, feature vectors for each image were extracted before normalization and response pooling operations, and calculated as the summed total activation of each kernel over the whole image (i.e. summing over the neurons). For fully-connected layers, the feature vector was simply the activations of each unit (no summing over the units required).

Analysis. First, to visualize the degree of similarity among views of different scales, we used multidimensional scaling, which projects the high-dimensional feature space into two dimensions, such that images that have more similar feature vectors are located closer together in space. Differences among images were computed using the Euclidean distance between feature vectors. Non-metric MDS was then performed over this distance matrix, projecting the data into 2D space for visualization.

To quantify this similarity, we used k-means clustering to group the images into 3 different categories based on their feature vectors extracted for each layer $(k=3$, squared Euclidian distance metric, 100 replicates). We evaluated how well the clustering solution matched the ground truth grouping of images by scale using the Rand Index. This index considers the set of pairwise comparisons between each of the images. Two images grouped together in both the clustering solution and the ground truth are counted as a correct pairing, and two items assigned to different groups in both the clustering solution and the ground truth are counted as a correct pairing. Grouping accuracy was then computed as the number of correct pairings divided by the total number of pairs, and multiplied by 100 to yield the grouping accuracy percentage. This outcome measure takes a value of 1 if the clustering solution perfectly agrees with the ground truth, but does not have a clear chance value. Thus, to estimate chance, simulations were run in which the grouping accuracy was computed when the object-reachspacescene labels were randomly shuffled over the image set. Chance was set as the mean grouping accuracy percentage over 1000 simulations. Finally, given that K-means clustering is a stochastic process, final clustering solutions depend in part on the locations of the randomly-seeded initial centroids, and the final Rand index is not a stable number. To converge on a more stable estimate of the grouping accuracy, the above clustering and scoring process was performed 100 times, and the average Rand index score is reported.

We also assessed the discriminability of the images using a Naive Bayes classifier with a leave-one-out cross validation training scheme. Features with zero variance were dropped prior to fitting the model, and feature distributions were modelled using a kernel density estimator. The classifier predicted whether a held-out image was an object, reachspace, or scene based on the feature activations, and classifiers were fit separately for each layer of ObjectNet and SceneNet, for both original and controlled images. We additionally performed several auxiliary analyses to test the discriminability of other divisions in the image set (see Table 1). All auxiliary 
analysis were conducted using the Naïve Bayes classifier and a leave-one-out cross validation scheme.

\section{Results \& Discussion}

First, we visualized the differences in deep net responses to objects, reachspaces, and scene images (Figure 5). Multidimensional scaling was used to project the high-dimensional feature space captured in each DNN layer into a 2D plot, such that points that are more distant in the plot have more dissimilar feature activations. The figure shows feature spaces derived from a network trained to discriminate objects categories (Figure 5a) and a network trained to discriminate scene categories (Figure 5b), for both original and controlled variants of the image set. There are three main observations that are evident in this visualization. First, object,

A. ObjectNet Object OReachspace Scene
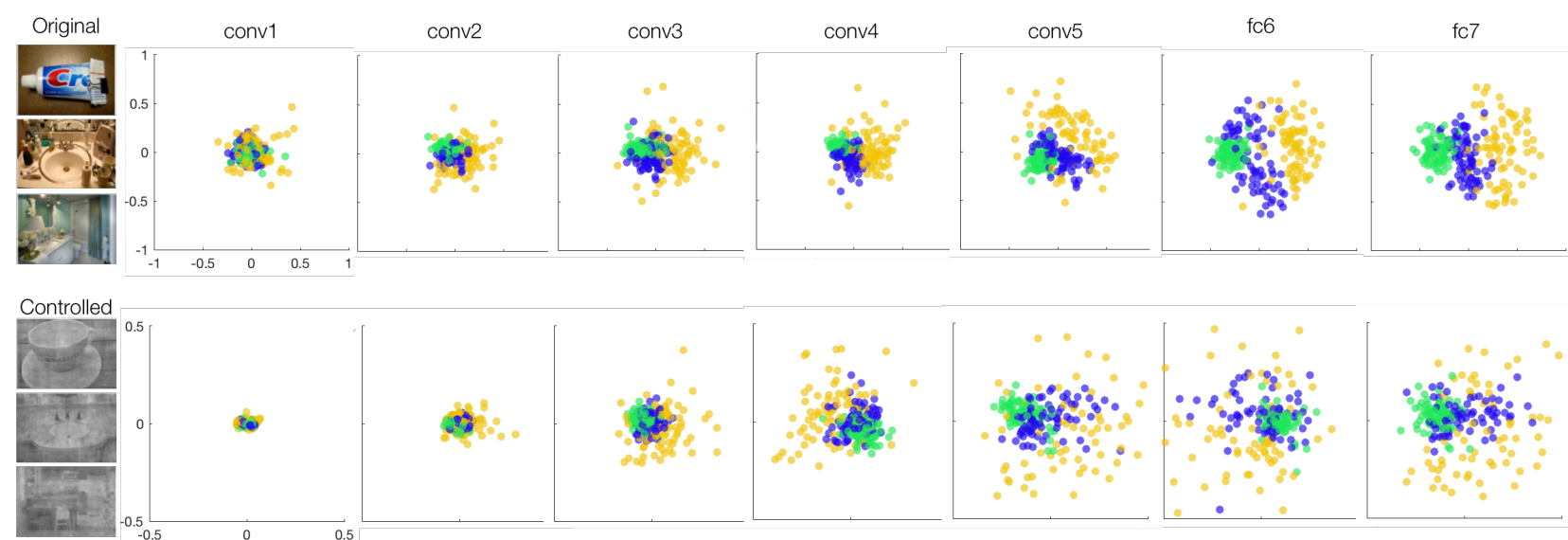

\section{B. SceneNet}

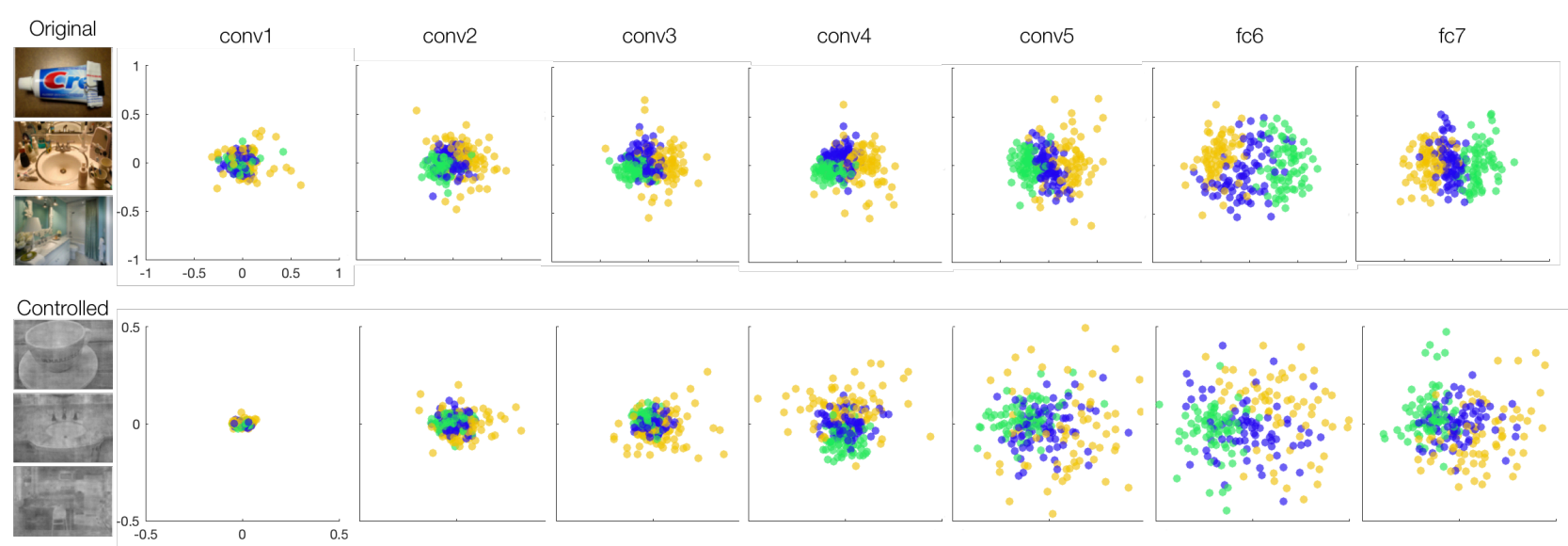

Figure 5. Multidimensional scaling plots of the feature similarity for objects (yellow dots), reachspaces (blue dots), and scenes (green dots). (A) Plots for each of the 7 layers of a deep net trained to do object recognition, where features were extracted from original images (top plot), and controlled images (bottom plot). (B). The same visualizations are shown as in (A) but for a network trained to classify scene categories. Note that the scale is different for the original image subplots compared to the controlled image subplots. The order in which data points were added to the graph was random, and data points are semi-transparent to enable clearer visualization of the distributions for all three scales of space 
reachspace, and scene images do have different feature activations in both networks, becoming increasingly distinct in later layers. Second, reachspace images are distinguished from both, and largely occupy intermediate positions relative to object and scene images. Third, these patterns also hold when images were equated in luminance and spatial frequency, particularly in later layers.

To quantify these observations, we used a data-driven clustering algorithm to divide the images into three clusters based on the similarity among their feature activations in each layer. Then, we calculated how well the data-driven clusters recovered the correct image classes (see Methods, Figure 4). Note that this data-driven method simply finds the major joints in the visual feature space; it does not need any training or labels. Thus, any cases where data-driven clusters correspond to the distinctions between image scales indicate that image scale is a major factor in the natural structure of the similarity space.

Results from the data-driven clustering analysis are shown in Figure 6, and confirm the patterns evident in the visualizations. That is, in ObjectNet, the different image scales were consistently assigned to separate clusters on the basis of their feature activations, in all layers beyond the lowest level feature representations of layer 1 (grouping accuracy for each successive layer: $55 \%, 66 \%, 76 \%, 73 \%, 71 \%, 87 \%$ and $77 \%$; simulated chance mean: $55 \%$ ). The same results held when image features were extracted from SceneNet (grouping accuracy for each layer: $55 \%, 63 \%, 71 \%, 77 \%, 67 \%, 86 \%$ and $74 \%$; simulated chance mean: $55 \%$ ). Further, when considering the feature activations to the controlled images, all layers beyond the first two showed this same natural grouping (grouping accuracy ObjectNet: 56\%, 54\%, 65\%, 62\%, 67\%, $67 \%$, and $66 \%$; SceneNet: $56 \%, 57 \%, 62 \%, 65 \%, 64 \%, 68 \%$, and $67 \%$; simulated chance mean: $55 \%)$.

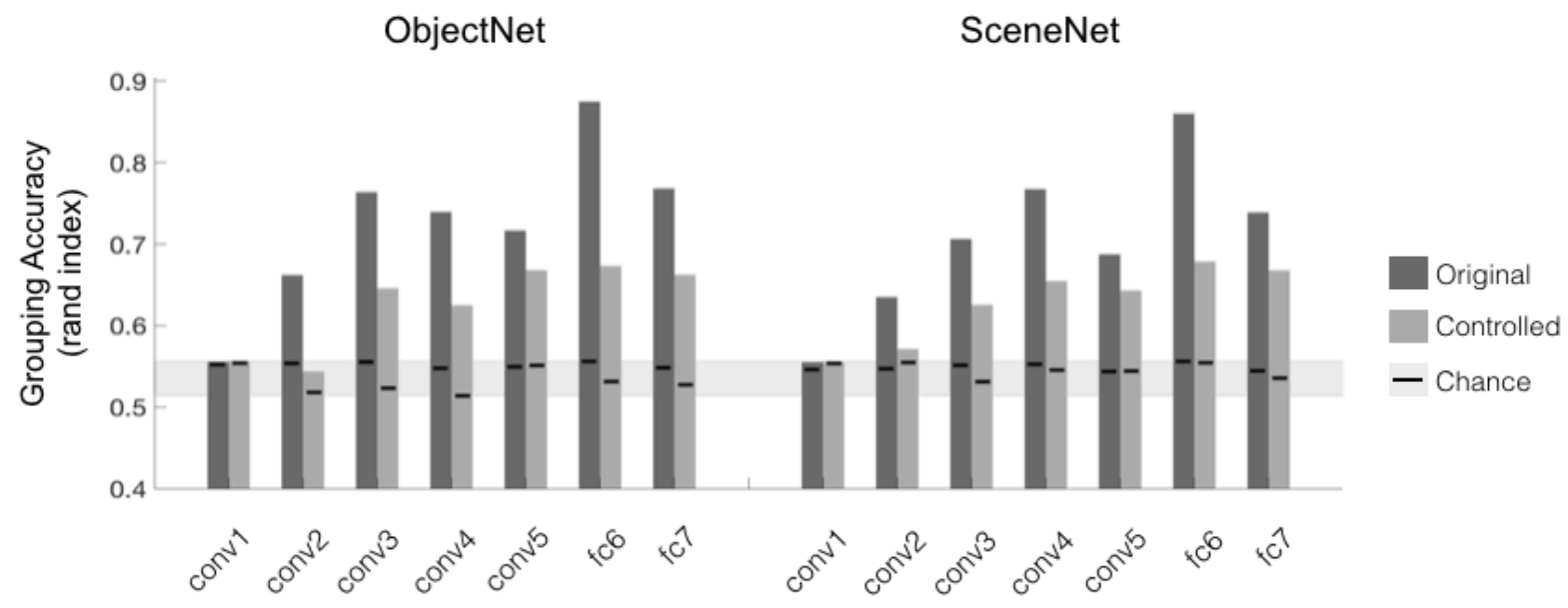

Figure 6. Grouping Accuracy results. The grouping accuracy score reflects how well the data-driven clusters recover the object, reachspace, and scene classes, quantified with the rand index. Grouping accuracy is plotted separately for ObjectNet (left) and SceneNet (right), for each layer, for both original images (dark gray bars) and controlled images (light gray bars). Chance was estimated separately for condition (small black horizontal line on each bar), with the range of chance values across these conditions depicted (light gray bar). 


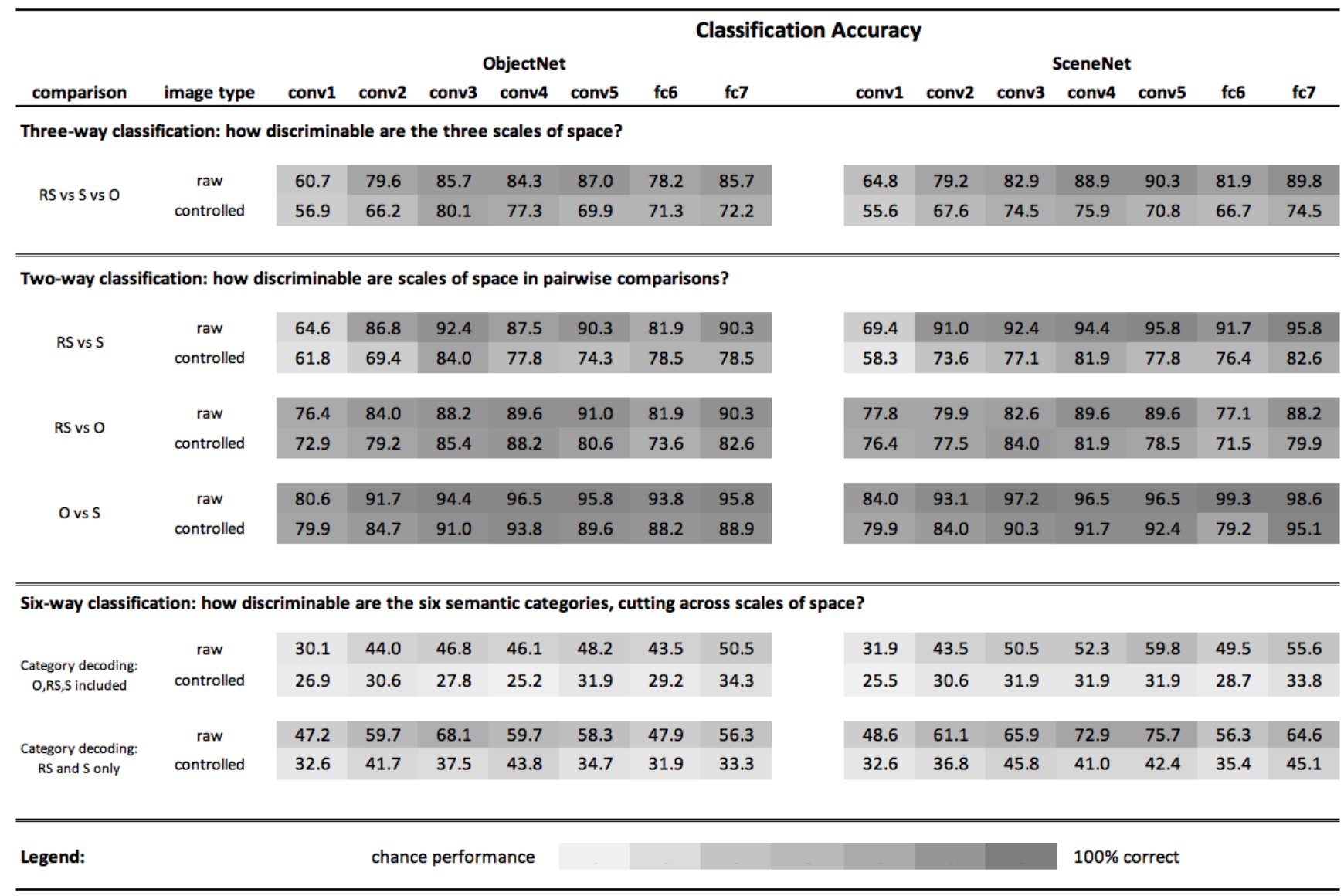

Table 1: Auxiliary results, using Naïve Bayes classification with leave-one-out cross-validation. The top panel reports the results of 3-way classification of the images into the three scales of space, for both raw and controlled images, in a network pre-trained to do object classification or scene classification (chance for this comparison was 33.3\%). The middle panel reports two-way classification accuracy, testing the discriminability of pairs of images (chance for these comparisons was 50\%). The bottom panel reports six-way classification accuracy for distinguishing among the 6 semantic categories, when considering all the images together or only the reachspaces and scenes together, for both raw and controlled images, in both networks (chance for these comparisons was $16.6 \%$ ). Cells in the table are shaded by their accuracy relative to chance for that particular comparison, with chance grouping performance in light gray, and $100 \%$ accuracy in dark gray.

To confirm that the results of the data-driven analysis generalized to other possible ways of analyzing the data, we also employed a classification approach (Table 1, top panel). A Naïve Bayes Classifier was trained to predict whether a held-out image was an object, reachspace, or scene based on the feature activations of each net and each layer. Cross-validated prediction accuracy was above chance in all layers. Note that the Naïve Bayes classifier did not show the performance boost for original images in layer fc6 that is apparent in the data-driven analysis and shown in Figure 6. However, this analysis generally showed a similar pattern of results to the data driven analysis: images can be classified by scale, and classification accuracy was highest in intermediate and later layers, for both ObjectNet and SceneNet, for both original and controlled image sets.

Finally, we additionally probed a number of other distinctions (Table 1). First, we considered whether objects, reachspaces, and scene feature differences would be evident in direct 2-way comparisons. For this analysis, we included only 2 scales of space, and used Naïve Bayes 
to assess how distinguishable pairs of scales are from each other. Overall, we find that objects and scenes are the most easily distinguished from each other, but that reachspaces remain highly dissociable from both scenes and objects in 2-way comparisons for both original images and controlled images. Second, we examined whether the six sematic categories were distinguishable from each other across in these feature spaces. We found that 6 -way classification was above chance by later layers, but overall was much less accurate than classification by scale of space. These results are consistent with the finding from E2 that perceptual differences between reachspaces and scenes were stronger than those for semantic category.

Taken together, these results primarily serve as an existence proof that there are image feature spaces in which reachspaces dissociate from both scenes and objects. However, these results also begin to provide some insight into the nature of the features that differentiate reachspaces from objects and scenes. First, given that grouping accuracy increases over layers, and that the correct grouping is still recovered when differences in luminance and spatial frequency are minimized, it is likely that features of mid-to-high level complexity underlie the divisions among scales of space. Second, the fact that reachspaces dissociated from other scales in both object-trained and scene-trained networks implies that this distinction does not rely solely on features specialized for distinguishing between specific object categories or specific scene categories. Third, the MDS visualization suggest that reachspaces occupy an intermediate position between objects and scenes in these feature spaces, further complementing the patterns in the behavioral data.

\section{General Discussion}

Research on the visual representation of the environment has proceeded largely by drawing a division between single objects and full-scale scenes, even though this may be an oversimplification of continuous visual experience (c.f. Henderson and Hollingworth, 1999). In the present study, we explicitly move away from this approach, and directly ask whether near-scale views of the environment, "reachspaces", are perceptually distinct from full-scale scene views, as well as singleton object views. Using human behavioral studies and deep neural networks, we found strong evidence for this dissociation. In Experiment 1 of the behavioral studies, visual search patterns showed a 3-way dissociation between objects, reachspaces, and scenes, for both luminance-matched and spatial-frequency-matched image sets. In Experiment 2, we showed that the perceptual difference between scenes and reachspaces was substantial: the magnitude of perceptual differences between scenes and reachspaces was much larger than the differences between scene categories in this image set. Finally, complementing these patterns in human behavior, we found that the features spaces learned by two different deep neural networks also naturally dissociate reachspace views relative to objects and scene views.

Taken together, the current study demonstrates that there are systematic feature distinctions between reachspaces and scenes, detectable by the human perceptual system, likely related to visual features of intermediate complexity. While it may be tempting to interpret this dissociation as evidence that reachspaces and scenes are separate mental categories, the present data do not support that inference, and future work will be required to explore this possibility. Rather, the current results point to the existence of systematic differences in perceptual content between scales of space previously treated as interchangeable. These results highlight that models of scene perception that do not distinguish between near and far space may be incomplete. In the following sections, we discuss the nature of the feature differences between reachspaces and 
scenes, the broader construct of reachspaces, and the implications that this perceptual division of space may have for our cognitive and neural architecture.

\section{The nature of the visual feature distinctions}

Given that reachspaces look systematically different from full-scale scenes, what are the visual features supporting this dissociation? We can draw some inferences about these features from the modeling results. Previous work has shown that in deep neural networks trained to do object or scene recognition, feature tuning increases in complexity over successive layers (Zeiler \& Fergus, 2014; Zhou, Khosla, Lapedriza, Xiao, Torralba \& Oliva, 2014; Guclu \& van Gerven, 2015), where layer 1 is dominated almost exclusively by representations of simple elements (oriented lines and colors); layers 2 and 3 see an increase in representations of textures and surfaces alongside the simple elements, and later layers (4 and 5) contain representations of object parts and full objects (Zhou et al., 2014). Given that all layers beyond the first one or two were sensitive to the object-reachspace-scene distinction, this suggests that mid-level features such as texture and surfaces, as well as high-level features such as object parts and entire objects may largely underlie the dissociation, while simple elements such as color, contrast and oriented lines do not.

There are also fundamental differences in the structure and constituent parts of reachspaces and scenes that may suggest other mid-level perceptual feature differences to explore. For example, reachspaces are dominated by small objects (e.g., bowls, sinks, pots) while scenes are dominated by large ones (e.g. tables, desks, rugs). Given that curvature varies with object size (Long, Konkle, Cohen, \& Alvarez, 2016; Long, Yu \& Konkle, 2018) it is possible that reachspaces have more curved contours while scenes have more rectilinear contours. Additionally, the reachspaces in this stimulus set all have a bounded surface on the bottom and are open on the top, while indoor rooms are enclosed on all sides. Thus, there are likely systematic differences in 3-dimensional layout features, and the spatial envelope of the views (Oliva \& Torralba, 2001). Finally, full-scene views encompass larger environments, and thus may include more elements and give rise to more perceptual clutter than reachspaces.

One open question is whether reachspaces are encoded in the visual system with distinct perceptual primitives. That is, are reachspaces processed by specific perceptual analyzers that are primarily dedicated to processing near-scale spaces? Or, are reachspace processed by weighted combinations of perceptual analyzers devoted to object-specific and scene-specific processing? One potential way to gain insight into this question is to leverage functional neuroimaging: if reachspaces drive some regions along the visual processing stream more strongly than both objects and scenes, this result would favor the possibility that reachspaces are not simply intermediate, and instead may have perceptual features of their own. However, regardless of the nature of the feature distinction, we have shown that for human perception, reachspace views are systematically different than scene views.

\section{Boundary conditions of reachspaces}

In the present work, we sampled our stimuli from what we think of as "canonical" reachspaces. That is, all reachspace views were of task-relevant near-scale spaces, largely within arm's reach, made up of semantically related objects arrayed on a horizontal surface, drawn from everyday contexts. However, there are other kinds of near-scale spaces with different 
characteristics. For example, bookshelves, pantries, ATMs and vending machines are spaces where we use our hands to manipulate objects, but they are primarily defined by a vertical plane. Likewise, photocopiers, ATMs and digital kiosks are made up of single large objects rather than multiple discreet objects. Would views of such non-canonical reachspaces still dissociate from scenes and objects, and would they group with more canonical reachspaces? Further, the intermediate perceptual status of our reachspaces relative to objects and scenes also raises an important question: do reachspaces reflect a point along a continuum from scenes to objects, characterized by smooth changes in perceptual features as the scale of space increases from objects to scenes, or are there more categorical boundaries where reachspaces reflect a distinct kind, characterized by abrupt changes in perceptual primitives from one scale to the next? Understanding the boundary conditions of what is a reachspace and understanding their categorical status are important new directions that arise from these results.

\section{Implications for cognitive and neural architecture}

The evidence for a perceptual dissociation, falling along functionally-relevant divisions of space, provides the foundation for future work exploring whether differences in neural and cognitive representations run alongside the perceptual differences reported here. Previous work has shown that patterns of neural activity in occipitotemporal cortex reflect low- and mid-level feature tuning (Groen, Ghebreab, Lamme \& Scholte, 2012; Groen, Silson \& Baker, 2017; Long, Yu \& Konkle, 2018; Watson, Young \& Andrews, 2016), as well as spatial layout and functional affordances (Park, Konkle \& Oliva, 2015, Bonner \& Epstein, 2017). Thus, it is possible that reachspaces will have distinct neural signatures along the ventral stream. Indeed, some previous evidence suggests that scene-processing areas can distinguish between near and far-scale spaces (Henderson, Larson \& Zhu, 2007; but see Epstein, Graham \& Downing, 2003). It is also likely that reachspace views engage cognitive processes that are not engaged by scenes and objects. For example, performing a task in a reachspace requires you to track the state of the task, the properties of the objects that signal the next step, the possible hand actions to be performed during that step (Hayhoe, 2000; Kirsh, 1995; Triesch, Ballard, Hayhoe \& Sullivan, 2003), while navigating through a scene requires you to track a very different set of attributes. Given that we spend most of our days in environments where objects are within reach and we are performing tasks, it is surprising that we still know very little about how these environments are represented. This study represents a step in extending our understanding of object and scene perception mechanisms to the perception of reachable space.

\section{Acknowledgments}

We wish to thank Bolei Zhou, and Chen-Ping Yu for their assistance with the computational modeling components, Chen Ping for building and training the CNNs from which we extracted visual features, Roger Strong for his help with sample size simulation, and Sarah Cohen for her assistance with data collection.

\section{Data Accessibility statements}

Stimuli and data for the behavioral experiments have been uploaded to an Open Science Framework repository (osf.io/7j6cx; Josephs \& Konkle, 2018) 


\section{References}

Biederman, I. (1987). Recognition-by-components: a theory of human image understanding. Psychological review, 94(2), 115.

Bonner, M. F., \& Epstein, R. A. (2017). Coding of navigational affordances in the human visual system. Proceedings of the National Academy of Sciences, 114(18), 4793-4798.

Bonner, M. F., \& Epstein, R. A. (2017). Computational mechanisms underlying cortical responses to the affordance properties of visual scenes. bioRxiv, 177329.

Brainard, D. H. (1997) The Psychophysics Toolbox, Spatial Vision 10:433-436.

Cadieu, C. F., Hong, H., Yamins, D. L., Pinto, N., Ardila, D., Solomon, E. A., Majaj, N.J. \& DiCarlo, J. J. (2014). Deep neural networks rival the representation of primate IT cortex for core visual object recognition. PLoS computational biology, 10(12), e1003963.

Carey, S., \& Xu, F. (2001). Infants' knowledge of objects: Beyond object files and object tracking. Cognition, 80(1-2), 179-213.

Carey, D. P., Dijkerman, H. C., Murphy, K. J., Goodale, M. A., \& Milner, A. D. (2006). Pointing to places and spaces in a patient with visual form agnosia. Neuropsychologia, 44(9), 15841594.

Cichy, R. M., Khosla, A., Pantazis, D., \& Oliva, A. (2017). Dynamics of scene representations in the human brain revealed by magnetoencephalography and deep neural networks. NeuroImage, 153, 346-358.

Cohen, M. A., Alvarez, G. A., Nakayama, K., \& Konkle, T. (2016). Visual search for object categories is predicted by the representational architecture of high-level visual cortex. Journal of neurophysiology, 117(1), 388-402.

Cowey, A., Small, M., \& Ellis, S. (1994). Left visuo-spatial neglect can be worse in far than in near space. Neuropsychologia, 32(9), 1059-1066.

Drew, T., Boettcher, S. E., \& Wolfe, J. M. (2016). Searching while loaded: Visual working memory does not interfere with hybrid search efficiency but hybrid search uses working memory capacity. Psychonomic bulletin \& review, 23(1), 201-212.

Duncan, J., \& Humphreys, G. W. (1989). Visual search and stimulus similarity. Psychological review, 96(3), 433.

Epstein, R. (2005). The cortical basis of visual scene processing. Visual Cognition, 12(6), 954978.

Epstein, R., Graham, K.S., Downing, P.E. (2003). Viewpoint-specific scene representations in human parahippocampal cortex. Neuron, 37. 865-876. 
Epstein, R., \& Kanwisher, N. (1998). A cortical representation of the local visual environment. Nature, 392(6676), 598.

Epstein, R., DeYoe, E. A., Press, D. Z., Rosen, A. C., \& Kanwisher, N. (2001). Neuropsychological evidence for a topographical learning mechanism in parahippocampal cortex. Cognitive neuropsychology, 18(6), 481-508.

Epstein, R., Graham, K. S., \& Downing, P. E. (2003). Viewpoint specific scene representations in human parahippocampal cortex. Neuron, 37(5), 865-876.

Gallivan, J. P., Cavina-Pratesi, C., \& Culham, J. C. (2009). Is that within reach? fMRI reveals that the human superior parieto-occipital cortex encodes objects reachable by the hand. Journal of Neuroscience, 29(14), 4381-4391.

Greene, M. R., \& Oliva, A. (2009). Recognition of natural scenes from global properties: Seeing the forest without representing the trees. Cognitive psychology, 58(2), 137-176.

Greene, M. R., \& Oliva, A. (2010). High-level aftereffects to global scene properties. Journal of Experimental Psychology: Human Perception and Performance, 36(6), 1430.

Grill-Spector, K., Kourtzi, Z., \& Kanwisher, N. (2001). The lateral occipital complex and its role in object recognition. Vision research, 41(10-11), 1409-1422.

Groen, I. I., Ghebreab, S., Lamme, V. A., \& Scholte, H. S. (2012). Spatially pooled contrast responses predict neural and perceptual similarity of naturalistic image categories. PLoS computational biology, 8(10), e1002726.

Groen, I. I., Greene, M. R., Baldassano, C., Fei-Fei, L., Beck, D. M., \& Baker, C. I. (2018). Distinct contributions of functional and deep neural network features to representational similarity of scenes in human brain and behavior. Elife, 7, e32962.

Groen, I. I., Silson, E. H., \& Baker, C. I. (2017). Contributions of low-and high-level properties to neural processing of visual scenes in the human brain. Phil. Trans. R. Soc. B, 372(1714), 20160102.

Güçlü, U., \& van Gerven, M. A. (2015). Deep neural networks reveal a gradient in the complexity of neural representations across the ventral stream. Journal of Neuroscience, 35(27), 10005-10014

Halligan, P. W., \& Marshall, J. C. (1991). Left neglect for near but not far space in man. Nature, 350(6318), 498.

Hayhoe, M. (2000). Vision using routines: A functional account of vision. Visual Cognition, 7(13), 43-64.

Henderson, J. M., \& Hollingworth, A. (1999). High-level scene perception. Annual review of psychology, 50(1), 243-271. 
Henderson, J. M., Larson, C. L., \& Zhu, D. C. (2008). Full scenes produce more activation than close-up scenes and scene-diagnostic objects in parahippocampal and retrosplenial cortex: an fMRI study. Brain and cognition, 66(1), 40-49.

Intraub, H., Bender, R. S., \& Mangels, J. A. (1992). Looking at pictures but remembering scenes. Journal of Experimental Psychology: Learning, Memory, and Cognition, 18(1), 180.

Intraub, H. (2010). Rethinking scene perception: A multisource model. In Psychology of learning and motivation (Vol. 52, pp. 231-264). Academic Press.

Josephs, E., \& Konkle, T. (2018, July 9). Perceptual dissociations among views of objects, scenes, and reachable spaces. Retrieved from osf.io/7j6cx

Jozwik, K. M., Kriegeskorte, N., Storrs, K. R., \& Mur, M. (2017). Deep Convolutional Neural Networks Outperform Feature-Based But Not Categorical Models in Explaining Object Similarity Judgments. Frontiers in psychology, 8, 1726.

Khaligh-Razavi, S. M., \& Kriegeskorte, N. (2014). Deep supervised, but not unsupervised, models may explain IT cortical representation. PLoS computational biology, 10(11), e1003915.

King, M., Groen, I. I. A., Steel, A., Kravitz, D., \& Baker, C. (2018). Similarity judgments and cortical visual responses reflect different properties of object and scene categories in naturalistic images. bioRxiv, 316554.

Kirsh, D. (1995). The intelligent use of space. Artificial intelligence, 73(1-2), 31-68.

Kleiner M, Brainard D, Pelli D, 2007, “What's new in Psychtoolbox-3?” Perception 36 ECVP Abstract Supplement.

Krizhevsky, A., Sutskever, I., \& Hinton, G. E. (2012). Imagenet classification with deep convolutional neural networks. In Advances in neural information processing systems (pp. 1097-1105).

Landis, T., Cummings, J. L., Benson, D. F., \& Palmer, E. P. (1986). Loss of topographic familiarity: An environmental agnosia. Archives of neurology, 43(2), 132-136.

Long, B., Konkle, T., Cohen, M. A., \& Alvarez, G. A. (2016). Mid-level perceptual features distinguish objects of different real-world sizes. Journal of Experimental Psychology: General, 145(1), 95.

Long, B., Störmer, V.S., \& Alvarez, G.A. (2017). Mid-level perceptual features contain early cues to animacy. Journal of Vision, 17(6), 1-20

Long, B., Yu, C.P, \& Konkle, T. (under review). A mid-level organization of the ventral stream. 
Maravita, A., \& Iriki, A. (2004). Tools for the body (schema). Trends in cognitive sciences, 8(2), 79-86.

Bertamini, M., Jones, L. A., Spooner, A., \& Hecht, H. (2005). Boundary extension: the role of magnification, object size, context, and binocular information. Journal of Experimental Psychology: Human Perception and Performance, 31(6), 1288.

Oliva, A., \& Torralba, A. (2001). Modeling the shape of the scene: A holistic representation of the spatial envelope. International journal of computer vision, 42(3), 145-175.

Oliva, A., \& Torralba, A. (2006). Building the gist of a scene: The role of global image features in recognition. Progress in brain research, 155, 23-36.

Palmer, E. M., Horowitz, T. S., Torralba, A., \& Wolfe, J. M. (2011). What are the shapes of response time distributions in visual search?. Journal of Experimental Psychology: Human Perception and Performance, 37(1), 58.

Park, S., Konkle, T., \& Oliva, A. (2014). Parametric coding of the size and clutter of natural scenes in the human brain. Cerebral cortex, 25(7), 1792-1805.

Pelli, D. G. (1997) The VideoToolbox software for visual psychophysics: Transforming numbers into movies, Spatial Vision 10:437-442

Potter, M. C. (1975). Meaning in visual search. Science, 187(4180), 965-966.

Potter, M. C. (1976). Short-term conceptual memory for pictures. Journal of experimental psychology: human learning and memory, 2(5), 509.

Ratcliff, R. (1979). Group reaction time distributions and an analysis of distribution statistics. Psychological bulletin, 86(3), 446.

Rousseeuw, P. J., \& Croux, C. (1993). Alternatives to the median absolute deviation. Journal of the American Statistical association, 88(424), 1273-1283.

Russakovsky, O., Deng, J., Su, H., Krause, J., Satheesh, S., Ma, S., Huang, Karpathy, A., Kholsa, A., Bernstein, M. \& Berg, A. C. (2015). Imagenet large scale visual recognition challenge. International Journal of Computer Vision, 115(3), 211-252.

Spelke, E. S. (1990). Principles of object perception. Cognitive science, 14(1), 29-56.

Steeves, J. K., Humphrey, G. K., Culham, J. C., Menon, R. S., Milner, A. D., \& Goodale, M. A. (2004). Behavioral and neuroimaging evidence for a contribution of color and texture information to scene classification in a patient with visual form agnosia. Journal of Cognitive Neuroscience, 16(6), 955-965.

Thorpe, S., Fize, D., \& Marlot, C. (1996). Speed of processing in the human visual system. nature, 381(6582), 520. 
Torralba, A., \& Oliva, A. (2002). Depth estimation from image structure. IEEE Transactions on pattern analysis and machine intelligence, 24(9), 1226-1238.

Torralba, A., \& Oliva, A. (2003). Statistics of natural image categories. Network: computation in neural systems, 14(3), 391-412.

Triesch, Jochen, Dana H. Ballard, Mary M. Hayhoe, and Brian T. Sullivan. What you see is what you need. Journal of vision3, no. 1 (2003): 9-9.

Võ, M. L. H., \& Wolfe, J. M. (2013). Differential electrophysiological signatures of semantic and syntactic scene processing. Psychological science, 24(9), 1816-1823.

Watson, D. M., Young, A. W., \& Andrews, T. J. (2016). Spatial properties of objects predict patterns of neural response in the ventral visual pathway. NeuroImage, 126, 173-183.

Willenbockel, Verena, Javid Sadr, Daniel Fiset, Greg O. Horne, Frédéric Gosselin, and James W. Tanaka. "Controlling low-level image properties: the SHINE toolbox." Behavior research methods 42, no. 3 (2010): 671-684.

Wolfe, J. M., Reijnen, E., Van Wert, M. J., \& Kuzmova, Y. (2009). In visual search, guidance by surface type is different than classic guidance. Vision research, 49(7), 765-773.

Wolfe, J. M. (2016). Visual search revived: the slopes are not that slippery: a reply to Kristjansson (2015). i-Perception, 7(3), 2041669516643244.

Wolfe, J. M., \& Horowitz, T. S. (2017). Five factors that guide attention in visual search. Nature Human Behaviour, 1(3), 0058.

Yamins, D. L., Hong, H., Cadieu, C. F., Solomon, E. A., Seibert, D., \& DiCarlo, J. J. (2014). Performance-optimized hierarchical models predict neural responses in higher visual cortex. Proceedings of the National Academy of Sciences, 111(23), 8619-8624.

Zeiler, M. D., \& Fergus, R. (2014, September). Visualizing and understanding convolutional networks. In European conference on computer vision (pp. 818-833). Springer, Cham.

Zhou, B., Lapedriza, A., Xiao, J., Torralba, A., \& Oliva, A. (2014). Learning deep features for scene recognition using places database. In Advances in neural information processing systems (pp. 487-495). 


\section{Appendix: sample size estimation}

All experiments were initially run with 12 subjects each, which in the past has been a standard sample size in visual search studies. However, among new efforts to increase reliability and replicability of results, reviewers requested that we add subjects in each experiment to increase power. To estimate a new target sample size, we used a simulation technique to estimate power over a range of sample sizes. We set the desired power value to $80 \%$ to detect the difference between Scenes-among-Scenes and Scenes-among-Reachspaces (one of our two critical effects).

In this simulation approach, hypothetical data sets for a range of sample sizes were constructed from previously obtained data. Specifically, for a given sample size, subjects were randomly sampled with replacement from the initial 12 subjects in the experiment. Trials were obtained for each of these simulated subjects by randomly sampling trials with replacement from the subject's original trials. The simulated dataset had the same number of trials per condition as the source dataset. Next, the average reaction time for each subject in each condition was calculated from this simulated dataset, and a paired t-test was performed for the critical comparison. This simulation was repeated 1000 times, and power was calculated as the percent of those simulations where $\mathrm{p}<0.05$. This process was repeated for each sample size ranging from 10 to 120 participants, and the lowest sample size that achieved at least $80 \%$ power was recorded

Applying this method, we obtained an estimated sample size of 28 from the E1a data, and 15 subjects from the E1b data. To derive a final sample size estimate that would be more robust to differences in variability between these two data sets, the samples size estimates were averaged over the two experiments, yielding a final sample size of 22 for both experiments. To estimate sample size for E2, which has fewer trials per condition than E1, we repeated the simulation, but sampled 30 trials per conditions during the trial-sampling step, matching the design of E2. Estimates were again derived from E1a and E1b data separately, and were averaged to yield a final sample size of 27 subjects for E2.

Following these analyses, additional data were collected to bring the total number of participants from $\mathrm{N}=12$ to $\mathrm{N}=22$ in both Experiment 1a and Experiment $1 \mathrm{~b}$, and to bring the total participants from $\mathrm{N}=12$ to $\mathrm{N}=27$ in Experiment 2. The overall patterns in the results were not changed by adding more participants, but all effects were substantially more robust. 